\title{
Effect of Electron Correlations on the Formation of Spiral Magnetic States in the Two-Dimensional $t-t^{\prime}$ Hubbard Model
}

\author{
P. A. Igoshev ${ }^{a, c}$, M. A. Timirgazin ${ }^{b}$, A. K. Arzhnikov ${ }^{b}$, and V. Yu. Irkhin ${ }^{a}$ \\ ${ }^{a}$ Institute of Metal Physics, Ural Branch, Russian Academy of Sciences, Yekaterinburg, 620990 Russia \\ ${ }^{b}$ Physical-Technical Institute, Ural Branch, Russian Academy of Sciences, Izhevsk, 426000 Russia \\ ${ }^{c}$ Ural Federal University, Yekaterinburg, 620002 Russia \\ e-mail: igoshev_pa@imp.uran.ru \\ Received June 14, 2013
}

\begin{abstract}
The formation of the spiral magnetic order in the ground state of the Hubbard model for a square lattice has been studied within the slave-boson method in a wide range of the parameters of the carrier density and Coulomb interaction. Next-nearest-neighbor electron hopping, as well as the magnetic phase separation, has been taken into account. It has been shown that the electron correlations lead to the suppression of the ferromagnetic and spiral phases in comparison with the Hartree-Fock approximation. The application of the results to the explanation of the experimental data, in particular, to the description of the magnetic state of the high-temperature superconductors of the cuprate-based systems has been discussed.
\end{abstract}

DOI: $10.1134 / \mathrm{S} 0021364013160054$

The investigation of the ground magnetic state of the single-band Hubbard model for already more than a half century has been an important and topical fundamental problem. In recent decades, the case of twodimensional lattices closely related to the problem of high-temperature superconductivity has been intensely studied. Conventionally, the ground state for the bipartite lattices is a Néel antiferromagnetic (AFM) insulator [1, 2].

The types of instability of the antiferromagnetic state in the presence of doping or the finite next-nearest neighbor hopping integral have still been incompletely revealed. According to the classical work by Nagaoka [1], when one charge carrier is added, the ground state on the bipartite lattice is the saturated ferromagnetic (FM) one. This statement can also be considered as a reasonable hypothesis in the case of finite doping $[1,3,4]$.

Scenarios of the possible doping-induced magnetic ordering include the phase separation of different types: to the ferromagnetic and antiferromagnetic phases [5] or the phase of the superconducting electron liquid and the Néel antiferromagnetic phase [6]. An alternative scenario is the formation of the spiral magnetic state. It was considered within different approaches: the analysis of the momentum dependence of the generalized static magnetic susceptibility for the bare spectrum [7], the Hartree-Fock approximation (small and moderate $U / W$ values, where $U$ is the parameter of the Coulomb repulsion and $W$ is the bandwidth) $[8,9]$, and the $t-J$ model (large $U / W$ values) [10].

Incommensurate structures are observed in doped high-temperature superconducting cuprates as the dynamic magnetic order [11], in the layered ceriumbased systems [12], and in iron-based high-temperature superconductors [13]. In addition, the considerably enhanced incommensurate magnetic fluctuations are observed in strontium ruthenates at low temperatures [14] (see discussion in [9, 15, 16]).

The study of the magnetic phase diagram of the two-dimensional Hubbard model taking into account the electron transfer only between the nearest neighbors $\left(t^{\prime}=0\right.$, where $t\left(t^{\prime}\right)$ is the integral of the transfer between the nearest (next-nearest) neighbors) within the Hartree-Fock approximation predicts that the spiral magnetic states are implemented in a wide range of parameters, especially at moderate values $U \lesssim W$ [17]. It was shown in [9] that the inclusion of the nextnearest neighbor electron transfer $\left(t^{\prime} \neq 0\right)$ into the Hamiltonian considerably changes the magnetic phase diagram of the ground state. The results obtained are in qualitative agreement with the experimental data for the magnetic structure of the layered high-temperature superconducting cuprates in the case of low doping (there are considerable quantitative discrepancies) [18].

The effect of the electron correlations on the stability of the spiral magnetic states using the slave-boson method was considered in [19]. The phase diagram of the Hubbard model was built in the nearest neighbor 
approximation $\left(t^{\prime}=0\right)$. Later, the generalized static magnetic susceptibility was studied within the same method. This makes it possible to determine the criterion of the instability of the paramagnetic state with respect to a second-order transition to the incommensurate magnetic state (which, in essence, generalizes the known criterion obtained within the random phase approximation [20] to the consideration of the strongly correlated states) [21]. As a result, a considerable tendency to ferromagnetic ordering at hole doping and a large $t^{\prime} / t$ value was found. However, the phase transitions between the magnetically ordered states cannot be studied within this approach.

In addition to the spiral structures, the formation of so-called stripes being the linearly polarized spin and charge density wave was studied [22]. It was established that they are more energy favorable than the spiral states at $t^{\prime} \neq 0$ and at a certain set of model parameters in the slave-boson approximation [23], as well as at $t^{\prime}=0$ and $U \leq W$ in the Hartree-Fock approximation [24]. However, this conclusion is somewhat devaluated by disregarding the intersite Coulomb interaction, which can considerably increase the energy of "stripes" as inhomogeneous structures.

It is known that the results of the angle-resolved photoemission for the high-temperature superconducting cuprates favor the inclusion of the next-nearest neighbor electron transfer "frustrating" the Néel antiferromagnetic order in the bare Hamiltonian [25]. Although the spiral states in the Hubbard model were actively studied, the relation between the type of magnetic order and the parameters of the problem (electron concentration $n, t^{\prime} / t, U$ ) has not been determined definitively. In addition, there is the question about the possibility of the formation of the inhomogeneous state in a wide sense: either by the magnetic phase separation or via the formation of stripes. In this paper, these questions are studied using the slave-boson method.

The slave-boson method in the form convenient for the study of the formation of the magnetic order was proposed by Kotliar and Ruckenstein [26]. In the saddle point approximation, this method is qualitatively close to the known Gutzwiller approximation. The energy of the ground state obtained within the slaveboson method is in good agreement with the quantum Monte Carlo and exact diagonalization calculations [19]. This justifies the usage of the slave-boson method in the cases where there are no strong fluctuations of the order parameter in the system. In the opposite case of the continuous (second-order) phase transition, where strong fluctuations occur, the nontrivial manyparticle renormalizations of Green's functions are needed indeed [15, 27].

The weakly modulated antiferromagnetic order competing with the Néel state is unstable near halffilling. As a result, a first-order transition takes place. In this case, it is possible to expect that the slave-boson method will give qualitatively reasonable results. This is favored by the comparison of the slave-boson approach and the dynamic mean field theory in the limit $U=\infty$ [28]. The error in the calculation of the spectral properties within the slave-boson method because of disregarding the local fluctuations slightly affects the determination of the energy. This makes it possible to consider this approach as a reasonable approximation.

We consider the two-dimensional Hubbard model for a spiral ordered state on the square lattice, where the matrix elements of the electron transfer $t_{i j}$ are $-t$ for the nearest neighbors and $t^{\prime}$ for the second neighbors. After the local rotation in the spin space matching the local magnetization vectors at different sites (which is necessary for the consideration of magnetic spirals) by the angle $\mathbf{Q R} \mathbf{R}_{i}$ (where $\mathbf{Q}$ is the wave vector of the spiral) and the introduction of the slave boson operators $e_{i}\left(e_{i}^{\dagger}\right), p_{i \sigma}\left(p_{i \sigma}^{\dagger}\right), d_{i}\left(d_{i}^{\dagger}\right)$, the action of the system can be described by the effective Hamiltonian, in which the Coulomb interaction has the form diagonal over the boson variables:

$$
\mathcal{H}_{\mathrm{eff}}=\sum_{i j \sigma \sigma^{\prime}} t_{i j}^{\sigma \sigma^{\prime}} f_{i \sigma}^{\dagger} f_{j \sigma^{\prime}} z_{i \sigma}^{\dagger} z_{j \sigma^{\prime}}+U \sum_{i} d_{i}^{\dagger} d_{i}
$$

where $f_{i \sigma}, f_{i \sigma}^{\dagger}$ are the slave Fermi operators, $t_{i j}^{\sigma \sigma^{\prime}}=$ $\exp \left[i \mathbf{Q}\left(\mathbf{R}_{i}-\mathbf{R}_{j}\right) \sigma^{x}\right]_{\sigma \sigma^{\prime}} t_{i j}$, and

$$
\begin{aligned}
z_{i \sigma}=(1 & \left.-d_{i}^{\dagger} d_{i}-p_{i \sigma}^{\dagger} p_{i \sigma}\right)^{-1 / 2}\left(e_{i}^{\dagger} p_{i \sigma}+p_{i \bar{\sigma}}^{\dagger} d_{i}\right) \\
& \times\left(1-e_{i}^{\dagger} e_{i}-p_{i \bar{\sigma}}^{\dagger} p_{i \bar{\sigma}}\right)^{-1 / 2} .
\end{aligned}
$$

The thermodynamic potential of the spiral state $\Omega(\mathbf{Q})$ is calculated in the saddle point approximation for the action $\mathcal{Y}$, introduced in [26] and generalized to the consideration of the spiral structures in [19] ( $z$ factors lose the dependence on $i$ and become $c$-numbers: $e_{i}, e_{i}^{\dagger} \longrightarrow e$, etc.). The conditions of the extremum of $\Omega$ with respect to the boson variables $e$, $p_{\sigma}, d$ and Lagrange factors lead to the system of the basic equations of the slave-boson method (see [19]). The resulting wave vector is determined by the minimization of $\Omega$ over various spiral states:

$$
\mathbf{Q}_{\mathrm{gs}}(\mu, U)=\arg \min _{\mathbf{Q}} \Omega(\mathbf{Q}, \mu, U) .
$$

The minimization of $\Omega(\mathbf{Q}, \mu, U)$ was performed numerically with a step of $0.02 \pi$ over $\mathbf{Q}$ within the following types of spiral states: $(Q, \pi)$ ("parallel" state), $(Q, Q)$ ("diagonal"), and $(0, Q)$. Since $\Omega$ in the ground state actually depends on the chemical potential $\mu$ as a parameter, we can determine the dependence of the magnetic structure on $\mu$ automatically taking into account the possibility of the phase separation [9]. 


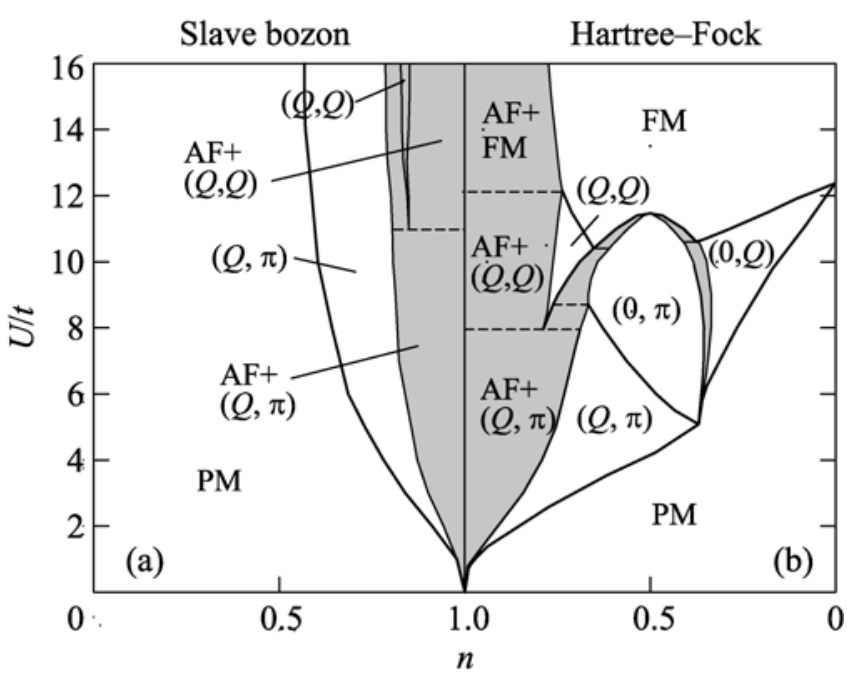

Fig. 1. Magnetic phase diagram of the ground state of the Hubbard model for $t^{\prime}=0$ at $n<1$ according to the (a) slaveboson method and (b) Hartree-Fock approximation. The phase diagram for the case $n>1$ due to the electron-hole symmetry $(n \longleftrightarrow 2-n)$ coincides with the given one. The spiral phases are denoted according to the form of their wave vector. Filling shows the regions of the phase separation. Bold lines denote the second-order phase transitions. Solid lines correspond to the boundaries between the regions of the homogeneous phase and the phase separation. Dashed lines show the regions of the separation of different phases.

The phase diagram for $t^{\prime}=0$ obtained within the slave-boson method is shown in Fig. 1a, where the phase transitions between the different magnetic states are first-order transitions that leads to considerable regions of phase separation. This considerably distinguishes this phase diagram from that presented in [19], where the phase separation was disregarded. The separation regions between the antiferromagnetic phase and the spiral magnetic states (parallel and diagonal) are especially wide. Therefore, the regions of the pure spiral states are narrowed. In particular, this refers to the diagonal phase, the existence of which becomes possible only at $U>11 t$. The phase transition between the paramagnetic and spiral magnetic states is a second-order transition.

Analogous phase diagrams of the ground state at different $t^{\prime} / t$ values were calculated within the Hartree-Fock approximation in [9] (for $t^{\prime}=0$, see Fig. 1b). It is seen that the electron correlations lead to the noticeable suppression of the magnetically ordered states in comparison with the Hartree-Fock approximation: the corresponding concentration intervals in the phase diagram decrease strongly, and the diversity of the spiral states disappears. The ferromagnetic state covering a considerable part of the diagram in the Hartree-Fock approximation is displaced to the region of large values $U \gtrsim 60 t$; this behavior reproduces the result obtained in [19] and is in good agreement with

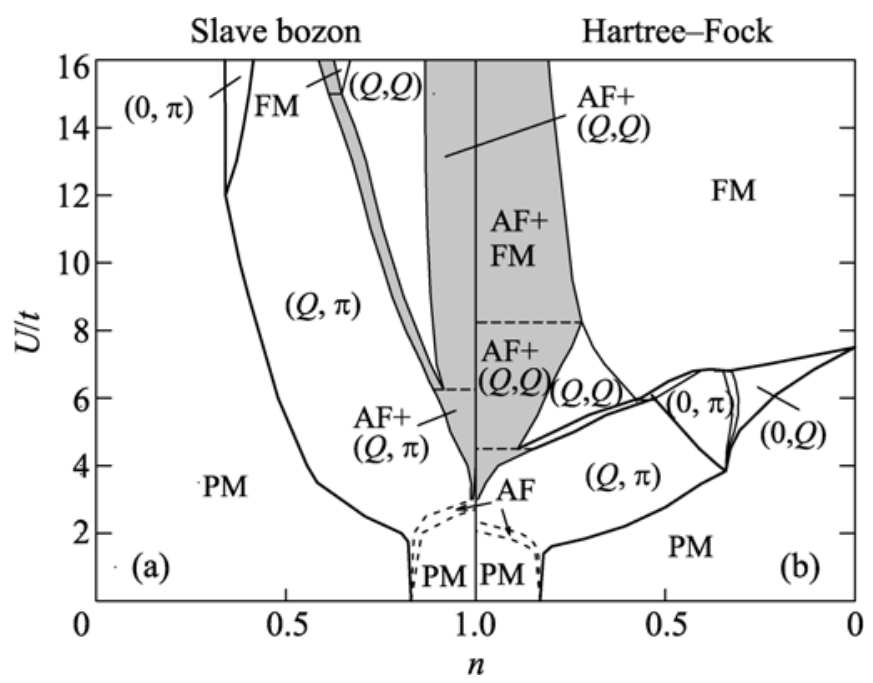

Fig. 2. Magnetic phase diagram at $t^{\prime} / t=0.2$ for $n<1$ according to the (a) slave-boson method and (b) HartreeFock approximation. Dashed lines denote the first-order phase transitions in the case where the region of the phase separation is narrow. The notation is analogous to that in Fig. 1.

the variational study of the stability of the saturated ferromagnetic phase [3]. The region of the separation of the antiferromagnetic and spiral phases narrows by approximately a factor of 2 .

According to our calculations, even the unlimited growth of $U$ does not make the magnetically ordered states stable far from half-filling: at $U=\infty$, there are no spiral magnetic solutions of equations of the slaveboson method at $n<0.37$ and $n>1.63$. At the same time, the saturated ferromagnetic solution becomes more favorable than the spiral ones at $|1-n|<0.3$. Thus, the spiral magnetic state at large $U$ values far from half-filling replaces the saturated ferromagnetic one. In contrast to [4], the unsaturated ferromagnetic solutions exist within our approach, but they are always energy unfavorable in comparison with the saturated ferromagnetic or spiral magnetic states.

It is known that the van Hove singularity of the electron spectrum (points $\mathbf{k}=(0, \pi),(\pi, 0))$ plays an important role in the formation of magnetism. At $U=0$, these points lie on the Fermi surface at a certain concentration $n_{\mathrm{vH}}$. At $t^{\prime}=0$, we have $n_{\mathrm{vH}}=1$ (half-filling), which singles out this case as a special one from the point of view of the magnetic properties. The deviation of $t^{\prime}$ from zero leads to the violation of the electron-hole symmetry and to the asymmetry of the phase diagram of the ground state.

Figure 2 presents the results obtained for $t^{\prime}=0.2 t$ at $n<1$ along with the Hartree-Fock approximation results. In comparison with the case $t^{\prime}=0$, the diagonal phase shifts to the region of much smaller $U / t$ values and the parallel phase becomes more extended over the concentration parameter. The physical origin 


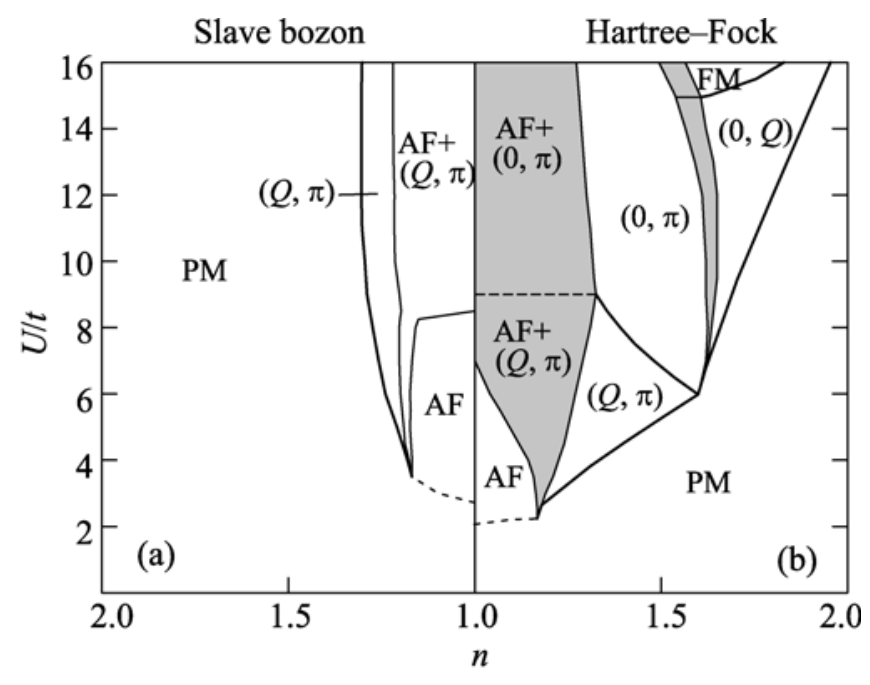

Fig. 3. Magnetic phase diagram at $t^{\prime} / t=0.2$ for $n>1$ according to the (a) slave-boson method and (b) HartreeFock approximation. The notation is analogous to that in Fig. 1.

is the detachment of the van Hove singularity $(n=$ $n_{\mathrm{vH}} \approx 0.83$ at $\left.t^{\prime}=0.2 t\right)$ and half-filling $(n=1$, one of the magnetic subbands is completely filled) at $t \neq 0$ giving their own tendencies to the magnetic ordering. It is seen that the correlation effects lead only to the quantitative renormalization of the phase boundaries.

In the case of $n>1$ (Fig. 3), the correlation effects are more significant: all homogeneous spiral states disappear except for a narrow region of the parallel phase. Such suppression of magnetism is explained by the fact that the only feature in this concentration interval is $n=1$. Far from half-filling, any spiral magnetism is impossible and the saturated ferromagnetism is unfavorable in comparison with the paramagnetic phase at any large $U$ value, when correlations are taken into account. Thus, the electron-hole asymmetry is enhanced considerably in comparison with the Hartree-Fock approximation, when correlations are taken into account.

These results are in agreement with the experimental data on the magnetic structure of the hole-doped compound $\mathrm{La}_{2-p} \mathrm{Sr}_{p} \mathrm{CuO}_{4}$, which has a close value of the $t^{\prime} / t$ parameter [29]. The neutron diffraction experiments showed that both the commensurate and incommensurate antiferromagnetic states at low hole doping $(p<0.02)$ are found in the $\mathrm{La}_{2-p} \mathrm{Sr}_{p} \mathrm{CuO}_{4}$ compound [30]. At $p \sim 0.02$, the system moves to the homogeneous incommensurate magnetic state with the wave vector $\mathbf{Q}=(\pi-\epsilon, \pi-\epsilon)$ (diagonal phase) [18], where $\epsilon$ increases approximately proportional to the doping level $p$ [31]. At $0.05<p<0.06$, the diagonal order coexists with the parallel incommensurate order with the wave vector $\mathbf{Q}=(\pi-\epsilon, \pi)$. At $p>0.06$, the transition to the parallel phase takes place [18]. The

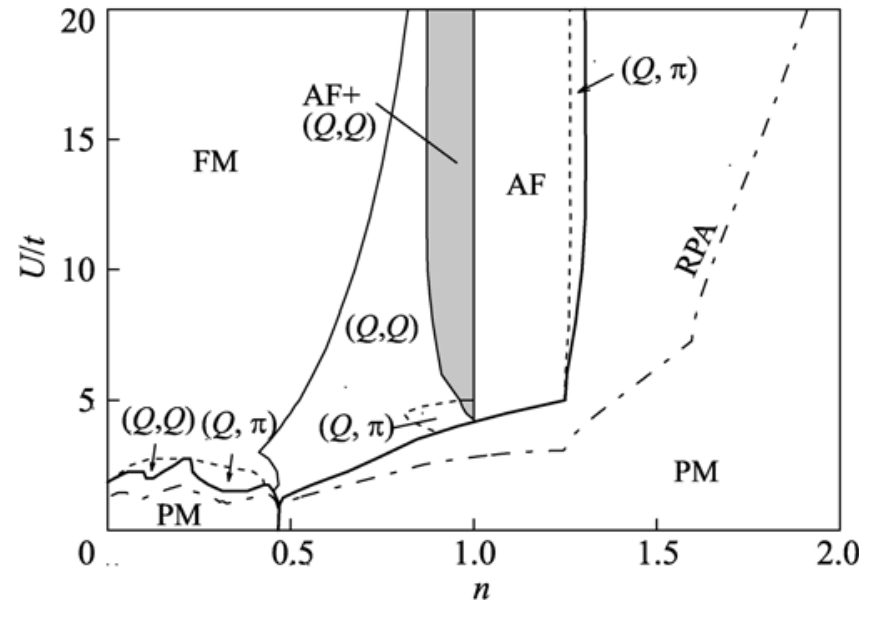

Fig. 4. Magnetic phase diagram at $t^{\prime} / t=0.45$. The dashdotted line is the phase boundary of the spiral and paramagnetic phases in the random phase approximation. The remaining notation is analogous to that in Fig. 2.

existence of the phase separation near half-filling is in agreement with the fact that the chemical potential is almost independent of doping at $0<p<0.1$ [32].

The sequence of phase transitions AFM $\rightarrow$ $\{\mathrm{AFM}+(Q, Q)\} \longrightarrow(Q, Q) \longrightarrow\{(Q, Q)+(Q, \pi)\} \longrightarrow$ $(Q, \pi)[18]$ observed in the experiment with increasing doping coincides with an analogous sequence in the diagram at $U \gtrsim 6.25 t$ (see Fig. 2). It is seen in the same figure that this sequence in the Hartree-Fock approximation takes place at $U \gtrsim 4 t$. Thus, the results of the slave-boson method are in agreement with the experimental data at the larger Coulomb repulsion parameters $U / t$ for $\mathrm{La}_{2-p} \mathrm{Sr}_{p} \mathrm{CuO}_{4}$. However, it is noteworthy that the experimental width of the region of the phase separation near half-filling between the antiferromagnetic and $(Q, \pi)$ phases is about 0.02 and the transition from $(Q, Q)$ to $(Q, \pi)$ state takes place at doping of 0.05-0.06. In our calculations, the concentration interval of the separation region $\mathrm{AFM}+(Q, \pi)$ is about 0.1 , which exceeds the actual value. The calculations showed that this value has a tendency to decrease with increasing $t^{\prime} / t$ parameter, but this decrease is insufficient to meet the experiment.

The incommensurate magnetic structure with the wave vector $\mathbf{Q}=(\pi-\epsilon, \pi)$ at $p>0.065$ was observed in the neutron scattering experiments in $\mathrm{YBa}_{2} \mathrm{Cu}_{3} \mathrm{O}_{7-y}$ (the charge carriers are holes) [33]. At the same time, for the high-temperature superconducting compound $\mathrm{Nd}_{2-x} \mathrm{Ce}_{x} \mathrm{CuO}_{4}$, in which the charge carriers are electrons, the homogeneous commensurate antiferromagnetic ordering is stable up to $x=0.14$ [34] in agreement with our results for $n>1$ (see Fig. 3).

Figure 4 shows the phase diagram for $t^{\prime}=0.45 t$. In comparison with the case $t^{\prime}=0$, here the regions of stability of the magnetic order at moderate $U$ and $n<1$ 
are considerably broadened due to the ferromagnetic and diagonal phases. The ferromagnetic state, which was absent at $t^{\prime}=0$ and moderately large $U$ values, becomes stable in this case already at small $n$ values and $U>3 t$. At $n>n_{\mathrm{vH}}$, the ferromagnetic and diagonal phases compete and the study of this competition apparently requires the accurate inclusion of nonlocal magnetic fluctuations [16, 27].

At $n>1$, the physical picture is completely different: the parallel phase is displaced by the commensurate antiferromagnetic phase, which remains almost the only phase in the region $n \lesssim 1.3$. This estimate for the "critical" carrier density is about the same for different $t^{\prime} / t$ values at $n>1$. Interestingly, with the unlimited increase in $U$, the concentration interval of the stability of the magnetic states is almost independent of $U: n_{c}(U=\infty) \approx 1.25$.

We also found the critical value $U_{c}^{\mathrm{RPA}, \mathrm{SB}}$ as the solution of the equation $1 / \max _{q} \chi_{q}(U, \omega=0)=0$, where the inhomogeneous susceptibility $\chi_{\mathbf{q}}$ was determined within the random phase approximation and in the slave-boson approach [21]. In both cases, $U_{c}$ values coincide with the corresponding boundaries of the second-order transition from the spiral to paramagnetic phase. At $n$ values close to $n_{\mathrm{vH}}$, the correlation renormalizations are small, while the ratio $U_{c}^{\mathrm{SB}} / U_{c}^{\mathrm{RPA}}$ increases at the deviation of $n$ from $n_{\mathrm{vH}}$ and this increase is faster for smaller $t^{\prime}$ values. This is due to the fact that a considerable fraction of the electron states at large $t^{\prime} / t$ values are concentrated near the van Hove singularity and the band bottom.

Thus, the correlation effects lead to the strong suppression of the regions of the existence of the magnetic phases. At the same time, the first-order transitions and the noticeable regions of the phase separation between the magnetically ordered states remain. The correlation effects near half-filling only slightly change the Hartree-Fock results, so at small values $t^{\prime} / t \lesssim 0.2$ they do not change the sequences of the magnetic states with increasing $U$.

The increase in the $t^{\prime} / t$ parameter leads to the redistribution of the electron density of states closer to the band bottom and to the van Hove singularity important for the formation of magnetism. In the slaveboson method, this leads to a much more considerable variation of the phase diagram than in the HartreeFock approximation: the asymmetry of the magnetic phases on the phase diagram with respect to the sign of the charge carriers increases. When the system is far from half-filling and the Fermi level is far from the van Hove singularity, the magnetic state cannot be formed at any $U$.

We are grateful to A.A. Katanin and M.V. Sadovskii for useful discussions. This work was supported in part by the Dynasty Foundation; by the Division of Physical Sciences and Ural Branch, Russian Academy of
Sciences (project nos. 12-T-2-1001, 12-T-3-1001); by the Presidium of the Russian Academy of Sciences (project nos. 12-P-2-1041, 12-U-2-1021); and by the Russian Foundation for Basic Research (project nos. 11-02-00931-a, 11-02-00937-a, 12-02-00632-a, and 12-02-31520-mol-a).

\section{REFERENCES}

1. Y. Nagaoka, Phys. Rev. 147, 392 (1966).

2. M. I. Katsnelson and V. Yu. Irkhin, J. Phys. C 17, 4291 (1984).

3. W. von der Linden and D. M. Edwards, J. Phys.: Condens. Matter 3, 4917 (1991); P. Wurth, G. S. Uhrig, and E. Müller-Hartmann, Ann. Phys. 2, 13960 (1997).

4. V. Yu. Irkhin and A. V. Zarubin, Phys. Rev. B 70, 035116 (2004); V. Yu. Irkhin and A. V. Zarubin, J. Exp. Theor. Phys. 43, 850 (2004).

5. P. B. Visscher, Phys. Rev. B 10, 943 (1973).

6. V. J. Emery, S. A. Kivelson, and H. Q. Lin, Phys. Rev. Lett. 64, 475 (1973).

7. H. J. Schultz, Phys. Rev. Lett. 64, 1445 (1990).

8. E. Arrigoni and G. C. Strinati, Phys. Rev. B 44, 7455 (1991).

9. P. A. Igoshev, M. A. Timirgazin, A. A. Katanin, et al., Phys. Rev. B 81, 094407 (2010).

10. B. Shraiman and E. Siggia, Phys. Rev. Lett. 62, 1564 (1990).

11. M. A. Kastner, R. J. Birgeneau, G. Shirane, and Y. Endoh, Rev. Mod. Phys. 70, 897 (1998).

12. W. Bao, P. G. Pagliuso, J. L. Sarrao, et al., Phys. Rev. B 62, R14621 (2000).

13. W. Bao, Y. Qiu, Q. Huang, et al., Phys. Rev. Lett. 102, 247001 (2009); D. K. Pratt, M. G. Kim, A. Kreyssig, et al., Phys. Rev. Lett. 106, 257001 (2011); Z. Xu, J. Wen, Y. Zhao, et al., Phys. Rev. Lett. 109, 227002 (2012); J. W. Lynn and P. Dai, Physica C 469, 469 (2009).

14. A. P. Mackenzie and Y. Maeno, Rev. Mod. Phys. 75, 657 (2003).

15. P. A. Igoshev, A. A. Katanin, and V. Yu. Irkhin, J. Exp. Theor. Phys. 105, 1043 (2007).

16. P. A. Igoshev, V. Yu. Irkhin, and A. A. Katanin, Phys. Rev. B 83, 245118 (2011).

17. S. Sarker, C. Jayaprakash, H. R. Krishnamurthy, and W. Wenzel, Phys. Rev B 43, 8775 (1991).

18. M. Fujita, K. Yamada, H. Hiraka, et al., Phys. Rev. B 65, 064505 (2002).

19. R. Fresard and P. Wölfle, J. Phys.: Condens. Matter 4, 3625 (1992).

20. T. Moriya, Spin Fluctuation in Itinerant Electron Magnetism (Springer, Heidelberg, 1985; Mir, Moscow, 1988).

21. R. Fresard and W. Zimmermann, Phys. Rev. B 58, 15288 (1998).

22. S. A. Kivelson, I. P. Bindloss, E. Fradkin, et al., Rev. Mod. Phys. 75, 1201 (2003). 
23. M. Raczkowski, R. Fresard, and A. M. Oles, Phys. Status Solidi B 244, 2521 (2007).

24. M. A. Timirgazin, A. K. Arzhnikov, and A. V. Vedyayev, Solid State Phenom. 190, 67 (2012).

25. A. Damascelli, Z. Hussain, and Z.-X. Shen, Rev. Mod. Phys. 75, 473 (2003).

26. G. Kotliar and A. E. Ruckenstein, Phys. Rev. Lett. 57, 1362 (1986).

27. A. A. Katanin and A. P. Kampf, Phys. Rev. B 68, 195101 (2003).

28. H. Park, K. Haule, C. A. Marianetti, and G. Kotliar, Phys. Rev. B 77, 035107 (2008).

29. T. Tanamoto, H. Kohno, and H. Fukuyama, J. Phys. Soc. Jpn. 62, 717 (1993).
30. M. Matsuda, M. Fujita, K. Yamada, et al., Phys. Rev. B 65, 134515 (2002).

31. K. Yamada, C. H. Lee, K. Kurahashi, et al., Phys. Rev. B 57, 6165 (1998).

32. A. Ino, T. Mizokawa, A. Fujimori, et al., Phys. Rev. Lett. 79, 2101 (1997).

33. V. Hinkov, D. Haug, B. Fauque, et al., Science 319, 597 (2008).

34. H. Takagi, S. Uchida, and Y. Tokura, Phys. Rev. Lett. 62, 1197 (1989).

Translated by L. Mosina 